\title{
Antagonism and synergy between extracellular BMP modulators Tsg and BMPER balance blood vessel formation
}

\author{
Jennifer Heinke ${ }^{1, \star}$, Maria Juschkat ${ }^{1}$, Anne Charlet $^{1,2}$, Leonie Mnich ${ }^{1}$, Thomas Helbing ${ }^{1}$, Christoph Bode ${ }^{1}$, \\ Cam Patterson ${ }^{3}$ and Martin Moser ${ }^{1}$ \\ ${ }^{1}$ Heart Center, Freiburg University, Cardiology and Angiology I, 79106 Freiburg, Germany \\ ${ }^{2}$ Department for Biology, Albert-Ludwigs University Freiburg, 79104 Freiburg, Germany \\ ${ }^{3}$ Division of Cardiology and McAllister Heart Institute, University of North Carolina at Chapel Hill, NC 27599-7075, USA \\ *Author for correspondence (Jennifer.Heinke@universitaets-herzzentrum.de)
}

Accepted 8 April 2013

Journal of Cell Science 126, 3082-3094

(c) 2013. Published by The Company of Biologists Ltd

doi: $10.1242 / j c s .122333$

\section{Summary}

Growth and regeneration of blood vessels are crucial processes during embryonic development and in adult disease. Members of the bone morphogenetic protein (BMP) family are growth factors known to play a key role in vascular development. The BMP pathway is controlled by extracellular BMP modulators such as BMP endothelial cell precursor derived regulator (BMPER), which we reported previously acts proangiogenically on endothelial cells in a concentration-dependent manner. Here, we explore the function of other BMP modulators, especially Tsg, on endothelial cell behaviour and compare them to BMPER. In Matrigel assays, BMP modulators chordin and noggin had no stimulatory effect; however, gremlin and Tsg enhanced human umbilical vein endothelial cell (HUVEC) sprouting. As the activation dynamics of Tsg were similar to those of BMPER, we further investigated the proangiogenic effect of Tsg on endothelial cells. Tsg enhanced endothelial cell ingrowth in the mouse Matrigel plug assay as well as HUVEC sprouting, migration and proliferation in vitro, dependent on Akt, Erk and Smad signalling pathway activation in a concentration-dependent manner. Surprisingly, silencing of Tsg also increased HUVEC sprouting, migration and proliferation, which is again associated with Akt, Erk and Smad signalling pathway activation. Furthermore, we reveal that Tsg and BMPER interfere with each other to enhance proangiogenic events. However, in vivo the presence of Tsg as well as of BMPER is mandatory for regular development of the zebrafish vasculature. Taken together, our results suggest that BMPER and Tsg maintain a fine-tuned equilibrium that controls BMP pathway activity and is necessary for vascular cell homeostasis.

Key words: BMP modulators, Angiogenesis, Endothelial cells, Signalling, Zebrafish

\section{Introduction}

During embryonic development the process of angiogenesis, i.e. the formation of new blood vessels from pre-existing ones, is highly important and tightly regulated by a multitude of signalling pathways (Heinke et al., 2012b; Herbert and Stainier, 2011). Many of the embryonic regulatory pathways have been found to be recapitulated during adult disease (Martin and Parkhurst, 2004; Sonnemann and Bement, 2011). Therefore, understanding embryonic molecular mechanism in angiogenesis provides further insight for therapeutic approaches under pathological conditions in the adult such as cancer, retinopathy, ischemic heart disease, stroke or pre-eclampsia (Carmeliet and Jain, 2011; Potente et al., 2011).

To generate a complex, intensively branched vasculature, endothelial cell behaviour has to be fine-tuned by a plethora of growth factors and signalling cascades to enable several cellular mechanisms including migration, proliferation and differentiation (Heinke et al., 2012b; Herbert and Stainier, 2011). For example, the well-known vascular endothelial growth factor (VEGF) activates the phosphoinositide 3-kinase (PI3K)/Akt signalling axis and the mitogen-activated protein kinase (MAPK)/ extracellular signal-regulated kinase (Erk) signalling cascades that in the following facilitate endothelial cell sprouting and survival (Muñoz-Chápuli et al., 2004; Olsson et al., 2006; Shiojima and Walsh, 2002). Besides VEGF, a lot of other different signalling mediators, such as bone morphogenetic proteins (BMPs), appear to cooperate in the many steps of angiogenesis (Adams and Alitalo, 2007; David et al., 2009; ten Dijke and Arthur, 2007).

BMPs belong to the transforming growth factor- $\beta$ (TGF- $\beta$ ) superfamily that have been extensively studied during embryonic development, in which they control axis formation and organogenesis (Wagner et al., 2010). BMPs are extracellular proteins that signal through cell surface complexes of heterodimeric transmembrane serine/threonine kinase receptors. Upon intracellular activation of the receptor, Smad $1 / 5$ transcription factors become phosphorylated and therefore obtain the ability to translocate to the nucleus where they modulate gene expression (Schmierer and Hill, 2007; Sieber et al., 2009). Besides this Smad-dependent pathway, we and others have shown that BMPs phosphorylate other Smad-independent signalling cascades such as MAPK/Erk and PI3K/Akt pathways 
(Bragdon et al., 2011; Sieber et al., 2009; Zhou et al., 2007). Accordingly, a growing body of evidence suggests that BMPs influence endothelial cell behaviour through activation of Smaddependent and Smad-independent pathways and along this line angiogenesis (David et al., 2009; Moreno-Miralles et al., 2009; Moya et al., 2012).

BMP signalling is highly regulated at the extracellular space by BMP modulators such as chordin (Piccolo et al., 1996), noggin (Smith and Harland, 1992), Drm/gremlin (Stabile et al., 2007), twisted gastrulation (Tsg) (Oelgeschläger et al., 2000) and BMP endothelial cell precursor derived regulator (BMPER) (Moser et al., 2003) (reviewed by Balemans and Van Hul, 2002; Umulis et al., 2009). BMPER, the vertebrate homologue of Drosophila crossveinless 2, is a secreted glycoprotein that contains five cysteine-rich domains followed by a von Willebrand D domain and a trypsin inhibitor domain and was originally identified in a screen for differentially expressed proteins in embryonic endothelial precursor cells (Moser et al., 2003). Previously, we and others have shown that BMPER may enhance BMP signalling in a concentration-dependent fashion (Heinke et al., 2008; Serpe et al., 2008). Lately, BMPER has been the subject of intensive research in the area of endothelial cell biology including inflammation (Helbing et al., 2010; Helbing et al., 2011), atherosclerosis (Pi et al., 2012a), tumour progression (Heinke et al., 2012a) and angiogenesis (Heinke et al., 2008; Moreno-Miralles et al., 2011). However, little is known about the role of BMP modulators chordin, noggin or Tsg in angiogenesis (David et al., 2009). As member of the BMP family Tsg contains the characteristic cysteine-rich domains that enable interaction between Tsg, BMPs and chordin (Yamamoto and Oelgeschläger, 2004). Similar to BMPER Tsg is reported to exert either pro- or anti-BMP effects depending on the examined model organism, developmental stage, organ or tissue (Chang et al., 2001; Little and Mullins, 2004; Nosaka et al., 2003; Oelgeschläger et al., 2000; Ross et al., 2001; Scott et al., 2001; Xie and Fisher, 2005; Zakin and De Robertis, 2004). Interestingly, recent studies in mice showed a genetic interaction between BMPER and Tsg in skeletal and nephron development indicating that they cooperate in fine-tuning BMP signalling (Ikeya et al., 2010; Ikeya et al., 2008; Zakin et al., 2008).

In this study we aimed to analyse and compare BMP modulators noggin, chordin, gremlin and Tsg in their ability to influence endothelial cell function in angiogenesis. We observed that BMP modulators chordin and noggin had no stimulatory effect, but gremlin and Tsg enhanced endothelial cell sprouting. Since gremlin has been investigated recently (Mitola et al., 2010; Stabile et al., 2007), we focused our interest on Tsg, which had the same activation dynamics of endothelial cell function as BMPER. Furthermore, we revealed interference between Tsg and BMPER to enhance proangiogenic events. However, in vivo the presence of Tsg as well as BMPER is mandatory for normal development of the zebrafish (Danio rerio) vasculature.

\section{Results}

Extracellular BMP modulators differently affect endothelial cell sprouting

In our previous work we reported BMPER to act proangiogenically on endothelial cells in a concentrationdependent manner (Heinke et al., 2008). In the present study, we aimed to investigate whether other known BMP modulators have similar effects on endothelial cell function. Therefore, we performed tube formation assays, and analysed different concentrations of BMP modulators chordin, noggin, gremlin and Tsg (Fig. 1). BMP modulators chordin and noggin had no stimulatory effect on HUVEC sprouting (Fig. 1A,C), formation of branch points (Fig. 1B,D), or transmigration through a modified Boyden chamber system (supplementary material Fig. S1). In contrast, high concentrations of chordin decreased HUVEC tube formation and transmigration to $79 \%$ compared to control conditions. Similar to the described concentrationdependent effect of BMPER gremlin and Tsg enhanced HUVEC sprouting by up to $57.4 \%$ (Fig. 1E) and $85.4 \%$ (Fig. 1G), respectively. Consistently, similar results are obtained by quantifying the number of branch points (Fig. 1F,H). Very recently, the angiogenic effect of gremlin has been investigated in detail by Presta's group (Mitola et al., 2010; Stabile et al., 2007). For this reason we focused our further research interest on the impact of Tsg on endothelial cell function.

\section{Tsg stimulates endothelial cell migration, proliferation and sprouting}

The in vitro Matrigel assay is a quick and easy method to investigate angiogenic endothelial cell behaviour such as reorganization of cytoskeleton and to search for cell-cell contact and cell fusion (Khoo et al., 2011; Ponce, 2009). Since we wanted to strengthen our basic findings on the Matrigel tube formation assay as a readout for angiogenic activity, we performed other in vitro angiogenesis assays such as transmigration, proliferation and spheroid sprouting assays. The effect of Tsg on endothelial cell migration was investigated by using a modified Boyden chamber system (Fig. 2A). To examine HUVEC proliferation a BrdU-based ELISA was used (Fig. 2B). In both assays Tsg increased endothelial cell migration and proliferation in a concentrationdependent manner. Similar to the results obtained from the tube formation assay (Fig. 1G,H) stimulation of HUVECs with Tsg in migration and proliferation assays resulted in a graph with a bellshaped curve. For 3D spheroid sprouting into a collagen matrix stimulation of HUVECs with low concentrations of Tsg had no effect, however at concentrations above $50 \mathrm{ng} / \mathrm{ml}$ sprout formation was significantly enhanced (Fig. 2C-E). Altogether, in vitro application of Tsg in several functional endothelial cell culture assays displayed a concentration-dependent proangiogenic effect.

\section{Tsg stimulates in vivo endothelial cell ingrowth in the mouse Matrigel plug assay}

To confirm our in vitro results that Tsg enhances endothelial cell sprouting, proliferation and migration in vivo we performed the mouse Matrigel plug assay (Fig. 3). Mice were injected subcutaneously with Matrigel alone or different concentrations of Tsg, sacrificed after 10 days and Matrigel plugs were embedded in paraffin. Application of Tsg increased the amount of ingrowing endothelial cells compared to unstimulated control plugs (Fig. 3A). To verify endothelial cell specificity a CD31$\mathrm{Cy} 3$ staining was performed that shows that the cellular ingrowth into the Matrigel plug consists mainly of endothelial cells as the majority of cells (nuclei stained blue) express the endothelial cell marker CD31 (stained in red) (supplementary material Fig. S2). Interestingly, quantification of mouse Matrigel plugs again revealed a bell-shaped curve of endothelial cell ingrowth in response to increasing concentrations of Tsg, indicating a complex mechanism by which $\mathrm{Tsg}$ regulates angiogenic processes (Fig. 3B). Taken together, these findings are 

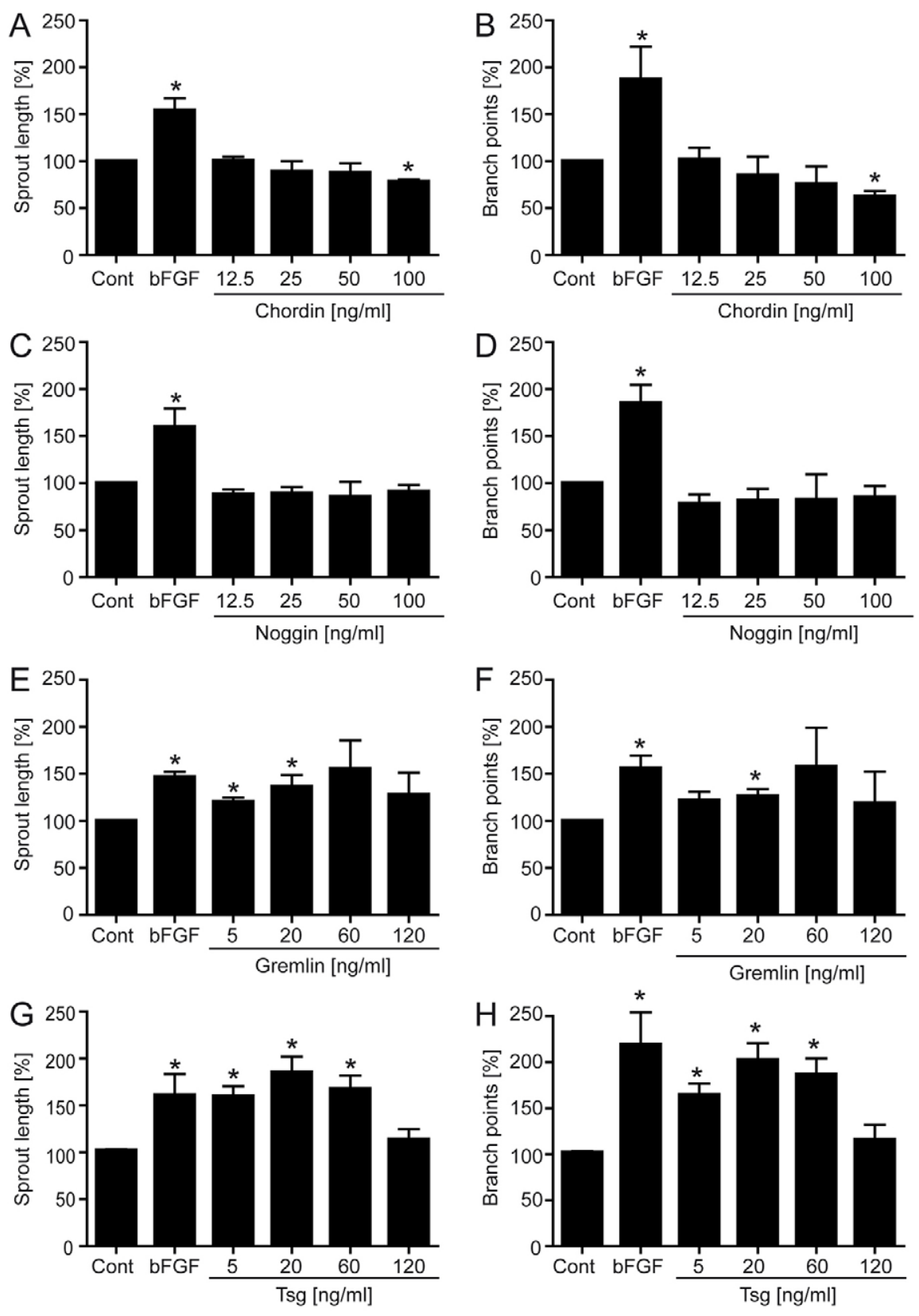

Fig. 1. Extracellular BMP modulators have different effects on endothelial cell sprouting in the tube formation assay. Serum-starved HUVECs were untreated or treated with bFGF $(50 \mathrm{ng} / \mathrm{ml})$ as a positive control, or with extracellular BMP modulators at the indicated concentrations, for 16-18 hours before they were seeded onto Matrigel with $2 \%$ FBS medium. Cumulative sprout length of capillary-like structures was measured after 3 hours. (A) Chordin, (C) noggin, (E) gremlin and (G) Tsg. In addition, the number of branch points was quantified for (B) chordin, (D) noggin, (F) gremlin and (H) Tsg. Values are means \pm s.e.m.; $n=3 ; * P<0.05$ versus control. consistent with our in vitro experiments and support the notion that Tsg has proangiogenic capacity and modulates endothelial cell function in a concentration-dependent manner.

\section{Tsg induces intracellular signalling pathways necessary for endothelial cell sprouting}

To investigate Tsg signalling pathways in endothelial cells we analysed Akt, Erk and Smad signalling cascades (Fig. 4A,B), because they have been implicated in angiogenic processes (Moya et al., 2012; Muñoz-Chápuli et al., 2004; Shiojima and Walsh, 2002). Indeed, stimulation of HUVECs with 10 to $20 \mathrm{ng} /$ $\mathrm{ml}$ Tsg protein increased Akt 1/2, Erk $1 / 2$ and Smad 1/5 pathway activation (Fig. 4A and supplementary material Fig. S3A-C). Time course experiments revealed that after 5 minutes of Tsg exposure all three signalling cascades are activated, however only Akt $1 / 2$ and Erk 1/2 phosphorylation persisted over time in different intensities (Fig. 4B and supplementary material Fig. S3D-F). To determine the impact of each signalling pathway on Tsg-induced endothelial cell sprouting, tube formation assays with pathway-specific inhibition were performed (Fig. 4C,E,G and supplementary material Fig. S4). First of all we ascertained concentrations for Erk inhibitors PD98059, U0126 and Akt inhibitor Akti VIII, which have a weak to none inhibitory effect compared to control conditions (data not shown). However, addition of inhibitors to Tsg-treated HUVECs prevented enhanced sprouting (Fig. 4C,E) and formation of branch points (supplementary material Fig. S4A,B). Western blot analysis of Tsg stimulated HUVECs with Erk $1 / 2$ and Akt 1/2 pathway inhibitors confirmed specific pathway inhibition (Fig. 4D,F). Furthermore, we observed that inhibition of Erk with PD98059 or U0126 increased Akt and Smad1/5 phosphorylation in parts conferred by total Smad protein stabilization (Fig. 4D). 

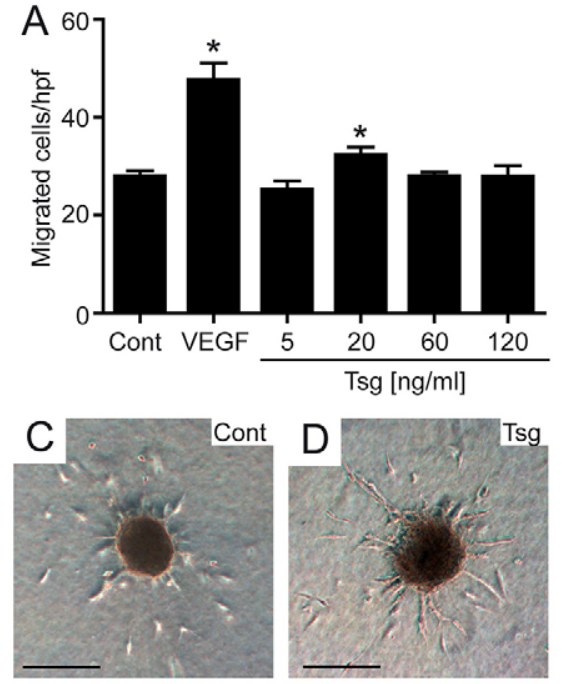

To ascertain if the observed Tsg effects are BMP dependent, BMP receptor II (BMPRII) expression was targeted with specific siRNAs. BMPRII knockdown efficacy was quantified by realtime PCR (supplementary material Fig. S4C). Indeed, deletion of BMPRII prevented Tsg-treated HUVECs from enhanced sprouting (Fig. 4G) and formation of branch points (supplementary material Fig. S4D). Of interest, in BMPRII siRNA treated cells Akt phosphorylation is increased, whereas Smad and Erk phosphorylation are unchanged (Fig. 4H). Stimulation with Tsg had no effect on BMPRII deficient cells, indicating a BMP pathway dependent effect of Tsg to stimulate intracellular signalling cascades. Together these data demonstrate that Tsg activates the Akt, Erk and Smad 1/5 signalling cascades,
Fig. 2. Tsg protein stimulates endothelial cell migration, proliferation and collagen gel spheroid sprouting. (A) For transmigration experiments HUVECs were serum-starved overnight and assayed with Tsg at the indicated concentrations or VEGF (100 ng/ml final concentration) as positive control, in migration medium containing $0.5 \%$ FBS. Triplicate samples were fixed after 4 hours and measurements were made on five random microscopic fields. Values are means \pm s.e.m.; $n=3 ; * P<0.05$ versus control. Hpf, high power field. (B) Proliferation was determined by a BrdU assay. Triplicate samples of HUVECs were incubated for 24 hours with BrdU and increasing concentrations of Tsg, or VEGF (100 ng/ml final concentration) as positive control, in medium containing $1 \%$ FBS. Cells in medium with BrdU only served as a negative control. Values are means \pm s.e.m.; $n=4$; $* P<0.01$ versus control. $(\mathbf{C}-\mathbf{E})$ HUVEC spheroids were embedded in collagen gel containing $10 \%$ FBS and stimulated for 24 hours with or without Tsg at the indicated concentrations, or with VEGF (50 ng/ml final concentration) as positive control. Representative spheroids incubated without (C) or with Tsg (D) are shown. Scale bars: $200 \mu \mathrm{m}$. (E) Quantitative analysis of cumulative sprout length of spheroids. Values are means \pm s.e.m.; $n=3 ; *=P<0.01$ versus control.
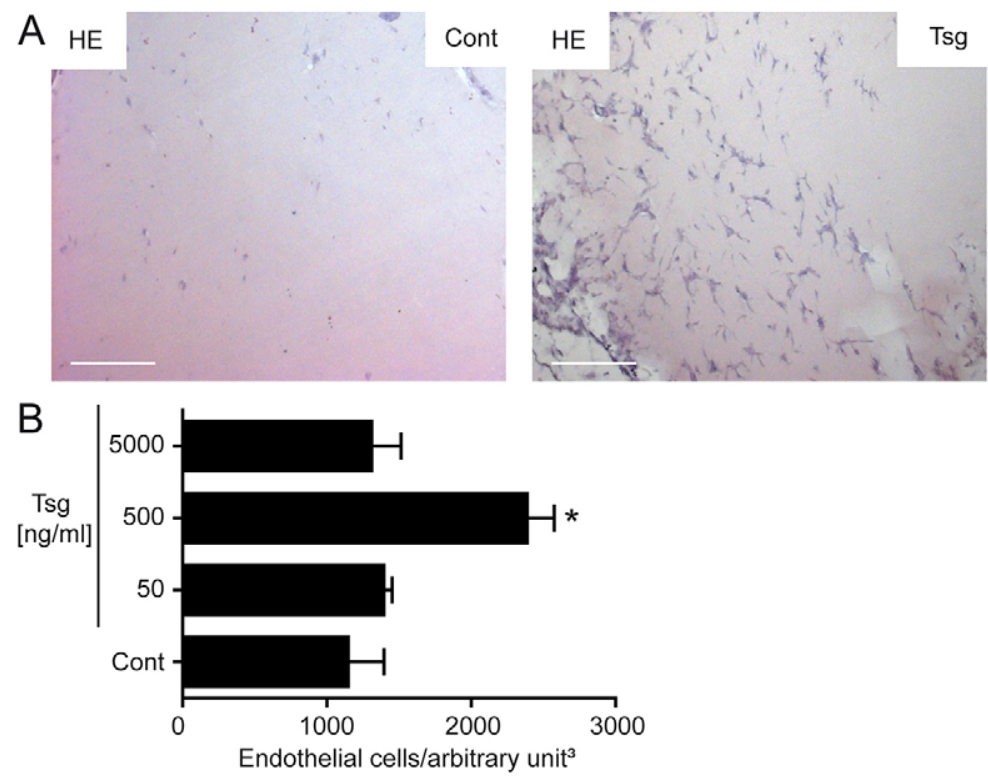

which have to act in concert to facilitate endothelial cell sprouting.

\section{Simultaneous application of Tsg and BMPER abolishes proangiogenic effects}

Recently, mouse genetic experiments revealed interactions of Tsg and BMPER during bone and kidney development (Ikeya et al., 2010; Ikeya et al., 2008; Zakin et al., 2008). Moreover, immunoprecipitation experiments showed a direct physical interaction between Tsg and BMPER (Ambrosio et al., 2008). Since Tsg displayed a similar concentration-dependent effect on endothelial cell sprouting as BMPER (Heinke et al., 2008) we next aimed to investigate combinatory effects of both in the tube 
A

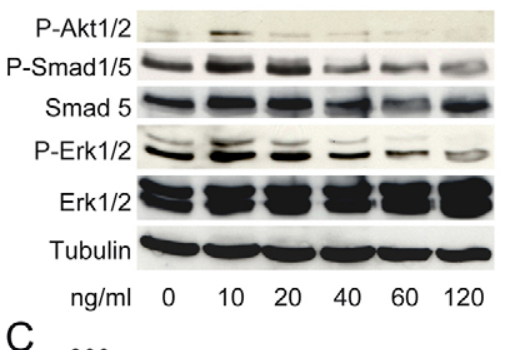

C

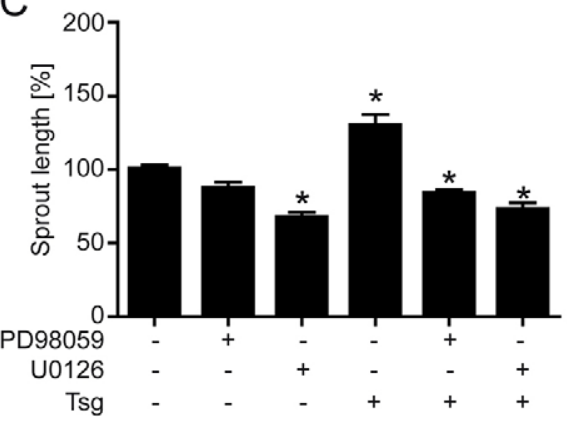

E

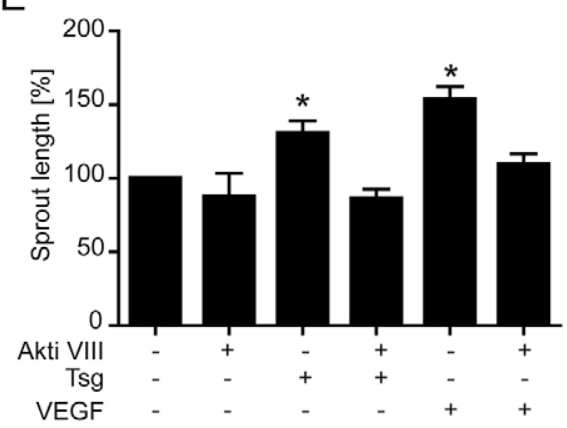

G

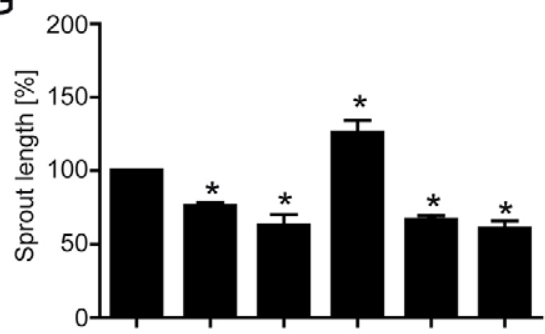

siRNA Cont BRII1 BRII2 Cont BRII1 BRII2

Tsg
B
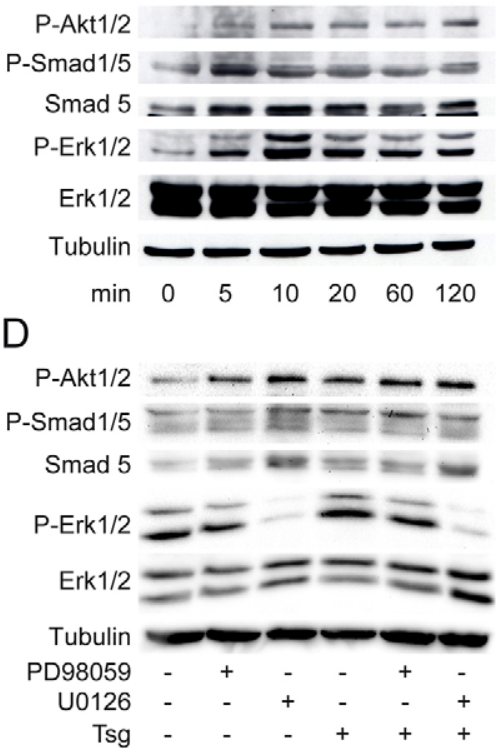

F

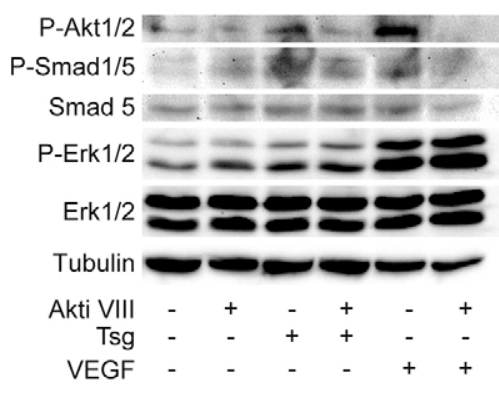

$\mathrm{H}$

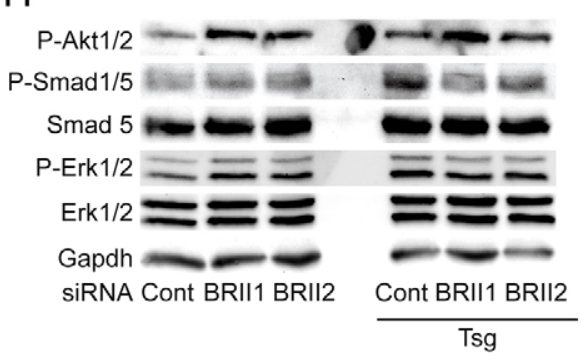

Fig. 4. Recombinant Tsg protein induces concentration- and time-dependent intracellular signalling necessary for endothelial cell sprouting. (A,B) Dose and time dependence of intracellular signalling by Tsg in HUVECs. Western blot analyses were performed with the indicated antibodies. (C,E) Cumulative sprout length of HUVEC capillary-like structures from tube formation assays. (C) Tsg-stimulated sprouting was inhibited when Erk was blocked with signalling pathway inhibitors PD98059 $(20 \mu \mathrm{M})$ and U0126 $(10 \mu \mathrm{M})$ or $(\mathrm{E})$ when Akt signalling was blocked with Akti VIII $(2 \mu \mathrm{M})$. Values are means \pm s.e.m.; $n=3 ; * P<0.01$ versus control. (D, F) Western blot analysis of Tsg-stimulated HUVECs with Erk inhibitors PD98059 $(20 \mu \mathrm{M})$ and U0126 $(10 \mu \mathrm{M})$ or Akt inhibitor Akti VIII $(2 \mu \mathrm{M})$ were performed with the indicated antibodies to determine the effects of inhibition of (D) Erk or (F) Akt phosphorylation on the other signalling pathways. (G,H) HUVECs were transfected with either of two BMPRII-specific siRNAs or scrambled siRNA control. (G) A tube formation assay was performed 48 hours posttransfection and cumulative sprout length was measured. Values are means \pm s.e.m.; $n=4$; ${ }^{*} P<0.01$ versus siRNA control. (H) 48 hours posttransfection cells were lysed and subjected to western blot analysis performed with the indicated antibodies. Representative western blots of one of three independent experiments are shown. formation assay in vitro and in the mouse Matrigel plug assay in vivo (Fig. 5). First we used the optimal concentration of Tsg to stimulate HUVEC sprout formation in the tube formation assay in vitro and added BMPER in equimolar amounts (Fig. $5 \mathrm{~A}-\mathrm{C}$ ). Application of BMPER and Tsg together prevented enhanced endothelial cell sprouting and branch point formation compared to Tsg alone, whereas at this high BMPER concentration endothelial cell sprouting was not affected. We confirmed these findings by analysis of intracellular signalling cascades (Fig. 5G). Vice versa, optimal concentrations of BMPER stimulated HUVEC sprouting and branch point formation and addition of equimolar amounts of Tsg, which at this concentration is below a stimulatory effect, inhibited the proangiogenic BMPER effect (Fig. 5D,E). We confirmed our in vitro findings in vivo with the mouse Matrigel plug assay (Fig. 5F). Again, co-stimulation with Tsg and BMPER abolished increased endothelial cell ingrowth compared to single protein application. Thus, Tsg and BMPER interfere with each other to enhance proangiogenic events in endothelial cells.

\section{Tsg and BMPER expression in endothelial cells of different origin}

As we have shown that Tsg and BMPER interact with each other, we asked if - besides BMPER - Tsg is expressed in endothelial cells. Indeed, Tsg and BMPER were detectable in human coronary arterial endothelial cells (HCAECs), venous endothelial cells (HUVECs) as well as in microvascular endothelial cells (HMECs) obtained from heart and lung (Fig. 6A-C). Furthermore, 
A
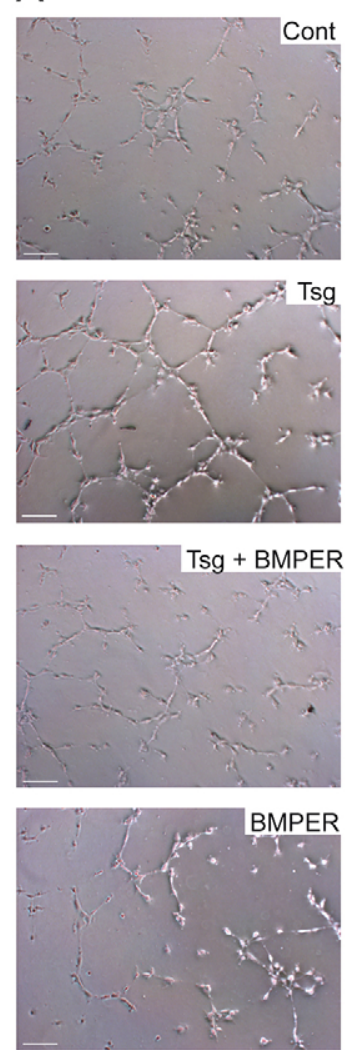

G

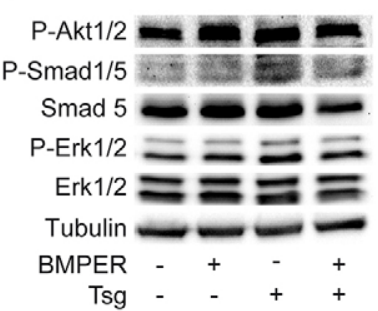

B

$\mathrm{D}$

$\mathrm{F}$
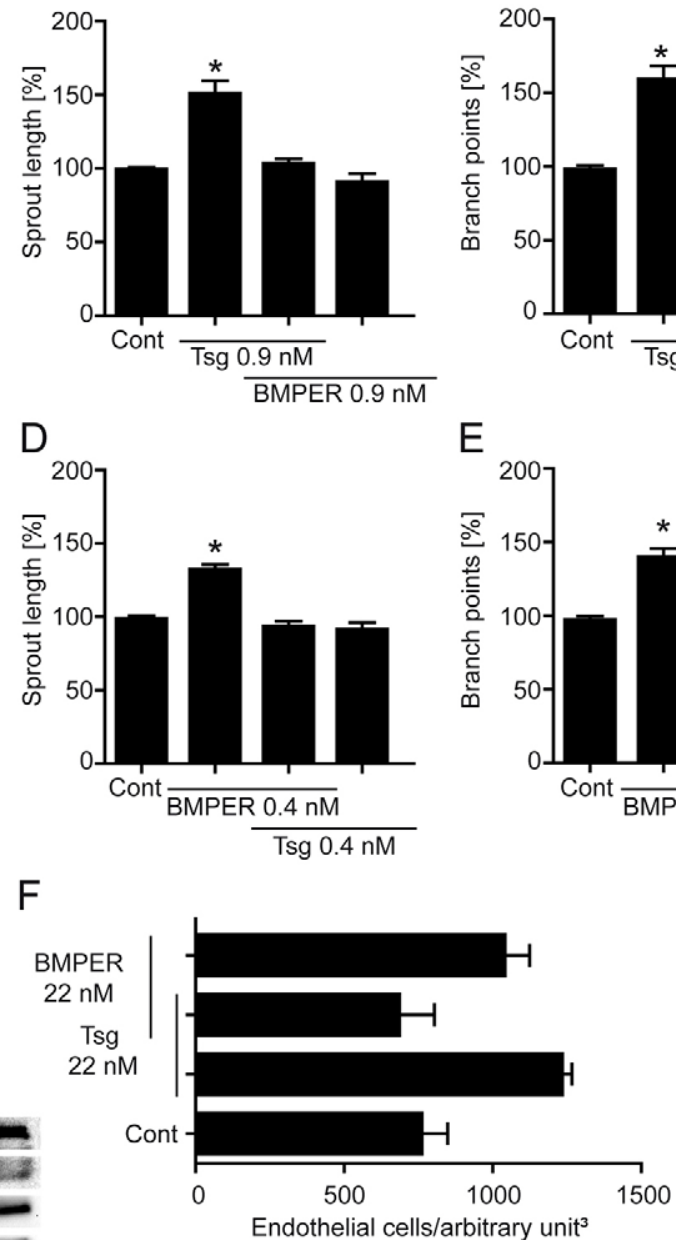

C

E
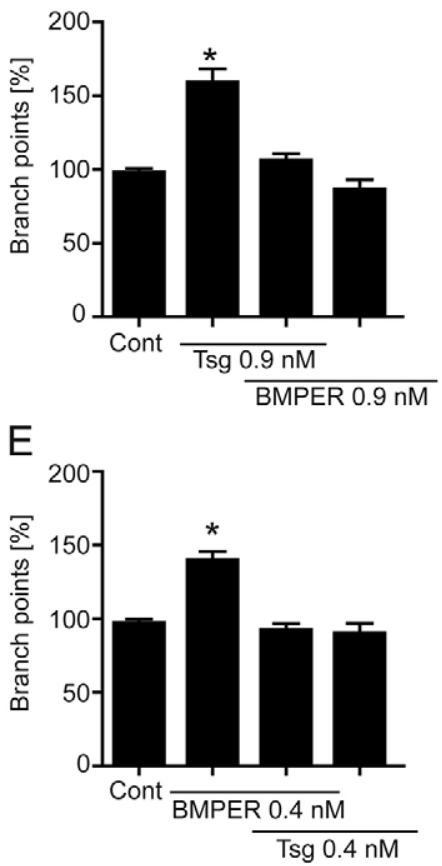

g. 5. Simultaneous application of Tsg and BMPER in functional in vitro and in vivo angiogenesis assays abolishes induction of proangiogenic effects. (A-C) Tsg-stimulated HUVEC sprouting was inhibited when recombinant BMPER protein was added in equal amounts. (A) HUVECs were incubated with the indicated proteins and subjected to a tube formation assay. Representative micrographs of control, Tsg, BMPER and Tsg + BMPER simultaneous application are shown. Scale bars: $200 \mu \mathrm{m}$. (B) Quantification of cumulative sprout length of capillary-like structures. (C) Number of branch points. (D,E) BMPER-stimulated HUVEC sprouting was inhibited when recombinant Tsg protein was added in equal amounts. (D) Quantification of cumulative sprout length of capillary-like structures. (E) Number of branch points. Values are means \pm s.e.m.; $n=3 ; * P<0.001$ versus control. (F) Quantification of mouse Matrigel plug assay with the indicated concentrations of recombinant Tsg and BMPER protein. Values are means \pm s.e.m.; $n=3$. (G) Western blot analysis of HUVECs stimulated with BMPER, Tsg and the combination of BMPER + Tsg (concentration $=0.9 \mathrm{nM}$ ) performed with the indicated antibodies. Representative western blots of one of three independent experiments are shown. we confirmed expression and localization of Tsg by immunocytochemistry in the cytoplasm and at the surface of HUVECs (Fig. 6D). Taken together, Tsg and BMPER are coexpressed by primary endothelial cells indicating that they per se play a role in endothelial cell biology.

\section{Inhibition of Tsg in endothelial cells enhanced sprouting and signalling}

Given that Tsg is expressed in endothelial cells we next aimed to investigate if loss of Tsg affects endothelial cell function. Therefore, knockdown efficiencies of two Tsg-specific siRNAs in HUVECS on mRNA and protein level were validated (supplementary material Fig. S5A-D). Based on the fact that stimulation with Tsg increased the angiogenic response we hypothesized that silencing Tsg would decrease sprouting, migration and proliferation of endothelial cells. However, the exact opposite was true: 3D collagen gel spheroid sprouting of Tsg-depleted HUVECs was increased compared to siRNA control transfected HUVECs (Fig. 7A,B). Along the same line, we detected enhanced migration (Fig. 7C) and proliferation capacity (Fig. 7D) of Tsg-silenced HUVECs. In the mosaic EC spheroid sprouting assay Tsg-depleted HUVECs (green fluorescent) formed most of the sprouts compared to equally mixed numbers of control siRNA-transfected cells (red fluorescent; Fig. 7E,F). Consistent with a proangiogenic effect, Tsg-silenced endothelial cells showed enhanced phosphorylation of Akt, Erk and Smad 1/5 signalling pathways (Fig. 7G). In summary, both stimulation with Tsg and depletion of Tsg increased angiogenic function. These data suggest that the concentration of Tsg is tightly balanced and controlled in endothelial cells.

\section{Balance of Tsg and BMPER in endothelial cell sprouting} We have shown that Tsg and BMPER block each other in increasing endothelial cells sprouting in the tube formation assay (Fig. 5). Here, we asked if stimulation with BMPER affects HUVEC sprouting when Tsg is silenced (Fig. 8A and supplementary material Fig. S5E) and vice versa, if stimulation with Tsg affects HUVEC sprouting when BMPER is silenced (Fig. 8B and supplementary material Fig. S5F). When Tsg was silenced HUVEC sprouting and branch point formation was increased compared to control, however, after additional 


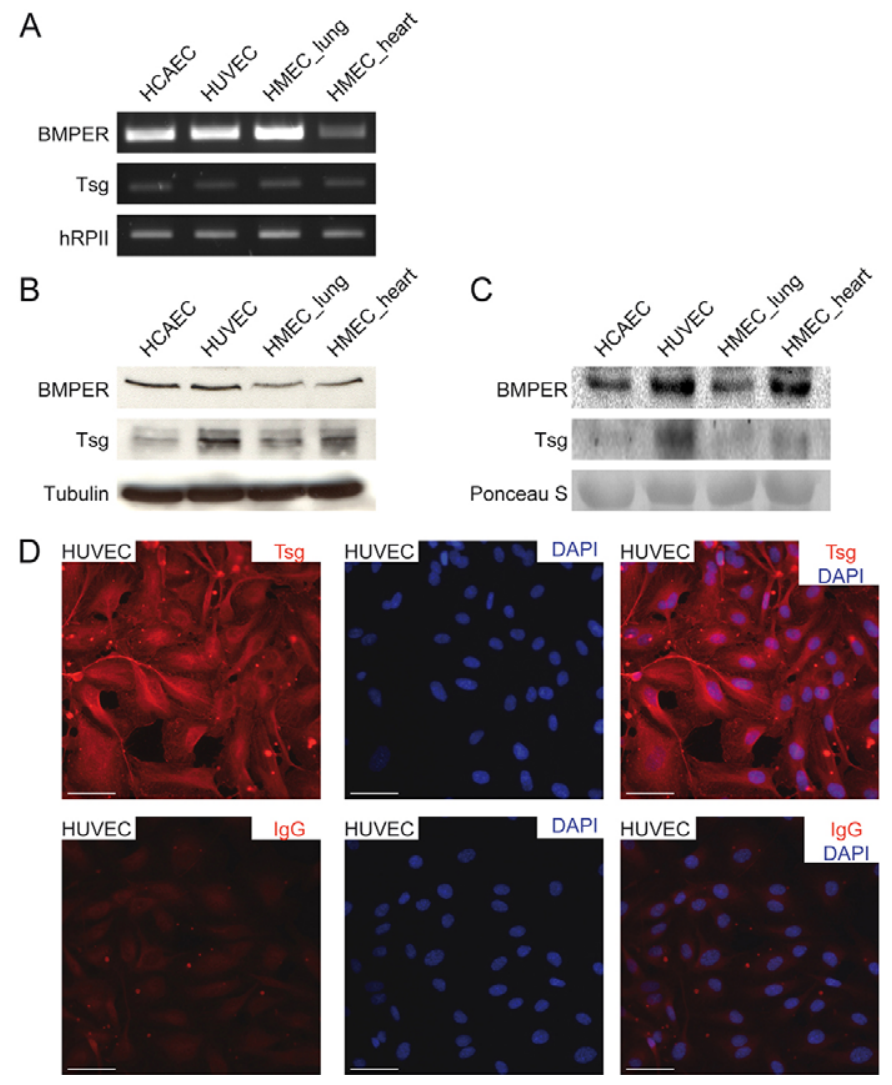

Fig. 6. Tsg and BMPER expression in endothelial cells of different origin. (A-C) Expression of Tsg and BMPER in human coronary artery ECs (HCAECs), HUVECs and human microvascular ECs (HMECs) from lung and heart were analysed by (A) RT-PCR, (B) western blot analysis of cell lysates and (C) supernatants. (D) Localization of Tsg protein by

immunocytochemistry in HUVECs (upper panel). Rat IgG2a was used as negative control (lower panel). Nuclei were stained with DAPI. Scale bars: $50 \mu \mathrm{m}$.

stimulation of Tsg-silenced HUVECs with BMPER no change occurred. On the other hand siRNA control-transfected HUVECs responded with increased sprouting to BMPER stimulation. BMPER-silenced HUVECs displayed decreased sprouting on Matrigel and after additional stimulation of BMPER-silenced HUVECs with Tsg again no change occurred. Together, these data indicate that Tsg and BMPER control each other to exert their angiogenic activity.

\section{BMPER and Tsg are necessary for proper angiogenesis in zebrafish development}

Next we investigated the in vivo relevance of Tsg and BMPER during development of the vasculature and therefore used the transgenic fli1:eGFP zebrafish model. In this zebrafish line the whole vasculature is fluorescent green because of the endothelialspecific activation of the ETS transcription factor Fli promoter sequences. We analysed the effect of morpholinos targeted against Tsg and BMPER on the formation of the intersomitic vessels (ISV) and caudal vein plexus (CVP) (Fig. 8C-E). Silencing of Tsg expression by morpholinos caused a large venous malformation (tail vein ectasia) with blood accumulation and some ISV defects (Fig. 8C,D). As already published before, silencing of BMPER resulted in several defects in ISV and disturbed formation of CVP (Moser et al., 2007) (Fig. 8D). Of interest, co-injection of Tsg morpholinos and BMPER morpholinos reduced the number of embryos that develop a tail vein ectasia (Tsg phenotype) but the number of embryos with ISV malformations was the same (BMPER phenotype; Fig. 8E). This observation might indicate that BMPER is epistatic to Tsg. Taken together the data suggest that the presence of Tsg as well as BMPER is mandatory for correct development of the vascular system in zebrafish.

\section{Discussion}

Only in recent years have BMPs been recognized to play an important role in the vasculature. In particular, how the different members of the BMP family influence endothelial cell function during processes of angiogenesis is of major interest (David et al., 2009; Moreno-Miralles et al., 2009; Pi et al., 2012b; Scharpfenecker et al., 2007; Zhou et al., 2007). Previously, we reported BMPER to act proangiogenic on endothelial cells in a concentration-dependent manner (Heinke et al., 2008). Here, we explore the function of BMP modulators noggin, chordin, gremlin and Tsg on endothelial cell behaviour and compare them to BMPER (Fig. 1). BMP modulators chordin and noggin had no stimulatory effect; however, gremlin and Tsg enhanced endothelial cell sprouting. As Tsg displayed the same activation dynamics as BMPER, we further investigated the proangiogenic effect of Tsg on endothelial cells in detail (Figs 2-4). Tsg enhanced endothelial cell ingrowth in the mouse Matrigel plug assay as well as HUVEC sprouting, migration and proliferation in vitro dependent on Akt, Erk and Smad signalling pathway activation in a strict concentration-dependent manner. Surprisingly, silencing of Tsg also increased HUVEC sprouting, migration and proliferation, which is again consistent with Akt, Erk and Smad signalling pathway activation (Fig. 7). Furthermore, we reveal that Tsg and BMPER interfere with each other to enhance proangiogenic events (Figs 5,6; Fig. 8A,B). However, in vivo the presence of Tsg as well as BMPER is mandatory for regular development of the zebrafish vasculature (Fig. 8).

In the past, extracellular regulation of BMPs has been described to occur only by antagonising proteins such as chordin and noggin, which inhibit BMP binding to receptors (Balemans and Van Hul, 2002). However, emerging evidence indicates that some BMP modulators, such as BMPER, exert both pro- and anti-BMP function (Moreno-Miralles et al., 2009). The situation becomes even more complex because some modulators, such as connective tissue growth factor (CTGF), have been shown to inhibit BMP signalling on the one hand and to promote TGF- $\beta$ signalling on the other (Abreu et al., 2002). Altogether, these findings led us to examine how different extracellular BMP modulators affect angiogenic function of endothelial cells. We determined three different functional groups of modulators. First, the classical BMP antagonists chordin and noggin that have no or at least an inhibitory effect on endothelial cell sprouting (Fig. 1A-D) and migration (supplementary material Fig. S1). Our results are consistent with reports from developmental studies on the notochord in which chordin and noggin, through BMP4 antagonism, led to reduced numbers of blood vessels (Nimmagadda et al., 2005; Reese et al., 2004). Secondly, we found BMP antagonist gremlin to exert proangiogenic effects on HUVECs (Fig. 1E,F), which recently Presta and co-workers reported to be independent of BMPs, but mediated by interaction with VEGFR2 (Chiodelli et al., 2011; Stabile et al., 2007). The 

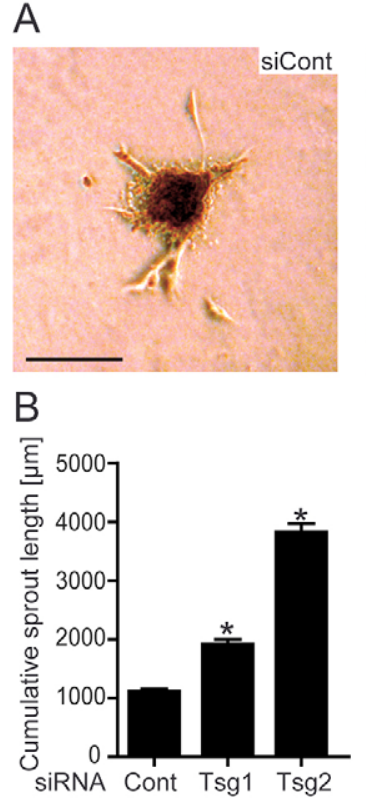

$\mathrm{E}$
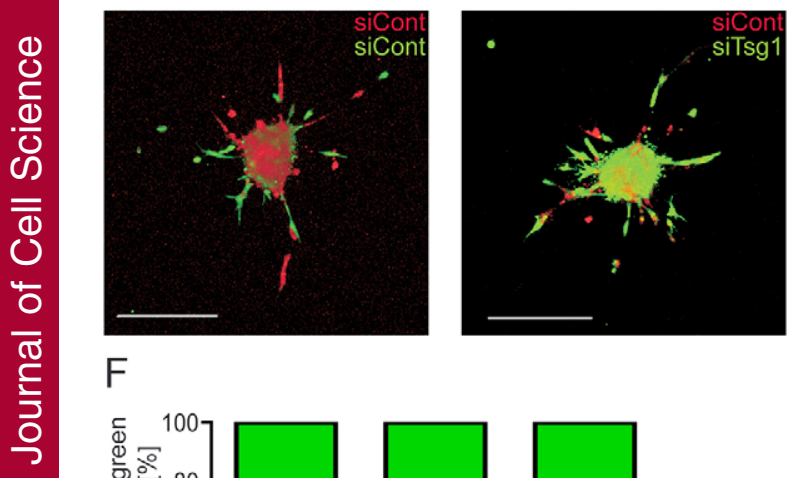

F

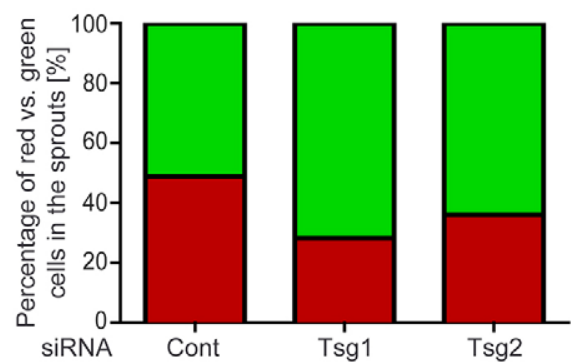

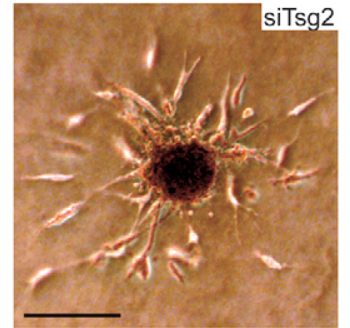

$\mathrm{D}$
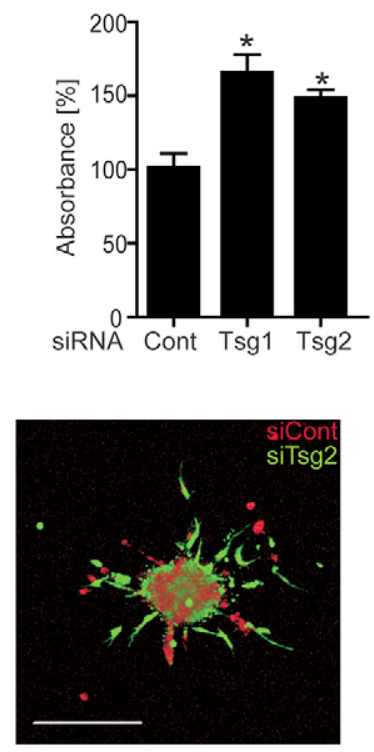

G

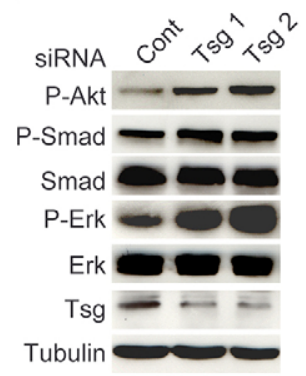

Fig. 7. Inhibition of Tsg in endothelial cells enhanced sprouting and signalling pathway activation. HUVECs were silenced for Tsg with either of two specific siRNAs or transfected with scrambled siRNA as control. (A,B) Six hours after siRNA transfection a collagen gel spheroid sprouting assay was performed. (A) Representative siRNAtransfected spheroids. Scale bars: $200 \mu \mathrm{m}$. (B) Quantitative analysis of cumulative sprout length of spheroids. Values are means \pm s.e.m.; $n=3 ; * P<0.001$ versus siRNA control. (C) Migration was quantified 48 hours post-transfection of HUVECs. Values are means \pm s.e.m.; $n=3 ; * P<0.05$ versus siRNA control. (D) Proliferation was determined by a BrdU assay. HUVECs were transfected in triplicate with the indicated siRNAs. 48 hours post-transfection BrdU ELISA was performed. Values are means \pm s.e.m.; $n=3 ; * P<0.001$ versus siRNA control. (E) Mosaic spheroids were generated by mixing equal amounts of HUVECs transfected with either siRNA control and labelled with CMPTX (red) or siRNA targeted against Tsg (or control) and labelled with CFDA-SE (green). Confocal laser microscopy revealed equally mixed green and red sprouts under control conditions, whereas in partially Tsg-silenced spheroids, Tsg-silenced cells predominantly formed sprouts. Scale bars: $200 \mu \mathrm{m}$. (F) Quantification of mosaic spheroids. (G) 48 hours after siRNA transfection cells were lysed and subjected to western blot analysis, performed with the indicated antibodies. third group consisted of BMPER and Tsg that activated angiogenic events in a concentration-dependent manner, which reached a peak and thereafter, at higher concentrations, diminished (Fig. 1G,H) (Heinke et al., 2008).

We and others recently discovered that BMPER acts in a concentration-dependent manner on BMPs and either activates or inhibits signalling (Heinke et al., 2008; Kelley et al., 2009; Serpe et al., 2008). Mechanistically, BMPER was shown to bind to different BMPs, type I BMP receptors and chordin, which dependent on stoichiometry and cellular context leads to pro- or anti-BMP effects (Ambrosio et al., 2008; Kelley et al., 2009; Serpe et al., 2008; Umulis et al., 2009). Of interest, Tsg is also found to either activate or inhibit BMP signalling (Little and Mullins, 2004; Xie and Fisher, 2005; Yamamoto and Oelgeschläger, 2004). Here, we have shown that in endothelial cells stimulation with Tsg leads to angiogenesis and activation of
Smad, Erk and Akt signalling pathways (Fig. 4). Consistently, these signalling cascades are well known to induce angiogenic responses (Moya et al., 2012; Muñoz-Chápuli et al., 2004; Shiojima and Walsh, 2002). BMPs are present in serum (Herrera and Inman, 2009) as well as endothelial cells produce and secrete endogenous BMPs (Heinke et al., 2008). To investigate the effect of Tsg on angiogenesis we used different experimental approaches, which contained different concentrations of serum. For example $2 \%$ serum was used for Matrigel tube formation assays, $0.5 \%$ serum for transmigration assays and serum-free Opti-MEM for stimulation of intracellular signalling cascades. Although quite different amounts of serum and along this line BMPs were present in the different assays, the Tsg effect is quite constant. However, the strength of the effect in the transmigration assay and the stimulation of intracellular signalling pathways are not as prominent as compared to the results of Matrigel tube 
formation and spheroid assays, which might be explained by the different serum concentrations - and thus BMP levels - in the different assays. Of interest, BMPER and BMP4 have been shown very recently to associate with low density lipoprotein receptor-related protein 1 (LRP1) and along this line acted proangiogenic on endothelial cell behaviour (Pi et al., 2012b). LRP1 is known to recognize more than 30 distinct ligands, cytoplasmic adaptor proteins and modulates the activity of other transmembrane receptors such as PDGFR- $\beta$ or TGF- $\beta$ R. Furthermore, LRP1 itself is known to activate Akt and Erk signalling pathways (Lillis et al., 2008; Muratoglu et al., 2010). Therefore, it is tempting to speculate that also BMP receptors, ligands and modulators form complexes with high signalling activity centres such as LRP1, which would localize the processing of incoming signals on the cell. Tsg might act in a similar way as - or interfere with - BMPER to enhance the angiogenic potential of endothelial cells. Taken together, this is the first report to determine that Tsg has a concentrationdependent proangiogenic effect on endothelial cells.

During mouse embryonic development, haemangioblasts develop in the aorto-gonadal-mesonephric (AGM) region, which subsequently give rise to endothelial capillary plexi around the whole embryo (Risau and Flamme, 1995). Of interest, BMP4, BMPER and Tsg are expressed in the AGM region indicating their role in endothelial cell biology (Marshall et al., 2000; Moser et al., 2003; Nosaka et al., 2003). To strengthen these findings, we confirmed expression of BMPER and Tsg in a variety of adult endothelial cells (Fig. 6).

Recent studies have demonstrated a physical interaction between Tsg, BMPER, chordin and BMP4 by immunoprecipitation (Ambrosio et al., 2008). Furthermore, genetic mouse models have shown interference between BMPER and Tsg to mediate BMP effects in the context of bone and nephron development (Ikeya et al., 2010; Ikeya et al., 2008; Zakin et al., 2008). In mice single homozygous gene deletions of Tsg or BMPER alone showed a decrease in ossification of vertebral bodies, but double deficient mice exhibited very severe reduction in ossification indicating a synergistic effect between BMPER and Tsg under these conditions (Ikeya et al., 2008). In the kidney, BMPER deficiency alone led to defects with lower nephron numbers, whereas in Tsg-deficient embryos the kidneys overall resembled the wild type. Of interest, Tsg and BMPER double-deficient mice displayed a wild-type phenotype indicating Tsg to be epistatic over BMPER in this context (Ikeya et al., 2010). During embryonic development, the kidneys develop by branching morphogenesis of a tubular system, which is a reoccurring theme during organ development and also found in lung and vascular development. Therefore, it is not surprising that similar molecular mechanism are involved to generate analogous morphological architecture (Herbert and Stainier, 2011; Horowitz and Simons, 2008). In our work we show that BMPER as well as Tsg are expressed in endothelial cells and that single application of recombinant BMPER or Tsg stimulate proangiogenic behaviour of endothelial cells (Figs 5, 6). This suggests that addition of certain concentrations of BMPER or Tsg to existent, endogenous complexes of BMP ligands and extracellular modulators such as chordin, BMPER and Tsg can evoke peak BMP signalling (Bier, 2008; Umulis et al., 2009). However, simultaneous application of equimolar concentrations of recombinant BMPER and Tsg abrogated the proangiogenic effect indicating that both proteins predominantly bind and therefore block each other to interact with endogenous BMP complexes in the extracellular matrix of endothelial cells. Along the same line, we hypothesize that after Tsg knockdown in endothelial cells the observed proangiogenic effect mimics the application of recombinant BMPER protein, because the balance between Tsg and BMPER shifted to more BMPER (Fig. 7). Our hypothesis is underlined by our finding that further addition of recombinant BMPER protein to Tsg-deficient endothelial cells led to no further increase in sprouting (Fig. 8A). Taken together, these data indicate that the concentrations of Tsg and BMPER have to be tightly balanced to enhance BMP signalling and along this line endothelial cell sprouting.

Previous in vivo experiments with Xenopus and zebrafish embryos have revealed the intriguing complexity of Tsg concentration- and context-dependent effects. High levels of Tsg overexpression causes ventralization of embryos, indicative of pro-BMP effects (Oelgeschläger et al., 2000; Ross et al., 2001), whereas low levels of Tsg overexpression causes dorsalization and therefore antagonizes BMP signalling (Chang et al., 2001; Ross et al., 2001; Xie and Fisher, 2005). On the other hand, injection of high doses of Tsg morpholino oligonucleotides (MOs) into zebrafish embryos causes dorsalization, indicating a pro-BMP effect of Tsg (Little and Mullins, 2004; Xie and Fisher, 2005). In contrast, low doses of Tsg MO causes ventralization and therewith indicate an anti-BMP-effect of Tsg (Ross et al., 2001; Xie and Fisher, 2005). Thus, the effect of Tsg seems to be strictly dependent on concentration. In addition, in zebrafish injected with low doses of Tsg MO all investigators noticed a ventral tail vein ectasia, where blood accumulates and the circulation slows (Little and Mullins, 2004; Xie and Fisher, 2005). Furthermore, Xie and Fisher observed an increase in gata 2 expression that is consistent with enhanced BMP signalling and haematopoiesis (Xie and Fisher, 2005). Moreover, BMP4 was shown to directly increase gata2 expression and along this line enhanced haemangioblast, haematopoetic and endothelial cells generation (Lugus et al., 2007). In our study with the transgenic zebrafish fli1:eGFP line, we again observed blood accumulation and the formation of an ectasia in the caudal vein plexus (CVP) after Tsg MO injection (Fig. 8C-E). We noticed a high density of green fluorescent endothelial cells in the developing CVP, which recently was shown to be dependent on BMP signalling (Wiley et al., 2011). Consistently, our Tsg deficiency data from zebrafish and HUVECs suggest that under these circumstances reduction of Tsg expression levels enhanced endothelial cell proliferation and sprouting by enhanced BMP signalling. On the contrary, BMPER morphants displayed a loss of CVP formation and in addition disturbed intersomitic vessel (ISV) growth (see also Moser et al., 2007) indicating loss of BMP signalling in the developing CVP. Of interest, in Tsg/BMPER double morphant embryos we observed less cyst formation compared to Tsg single morphants, suggesting that the cyst formation is caused by BMPER and enhanced BMP signalling in the absence of Tsg. In contrast, the ISV sprouting seems to be independent of Tsg, as in double morphants the phenotype of single BMPER morphants persist. This is consistent with our BMPER-depleted HUVEC Matrigel assays, where in the absence of BMPER Tsg is unable to induce sprouting. Taken together, in angiogenic events Tsg seems to be dependent on BMPER, whereas loss of BMPER also displayed Tsg-independent effects. 


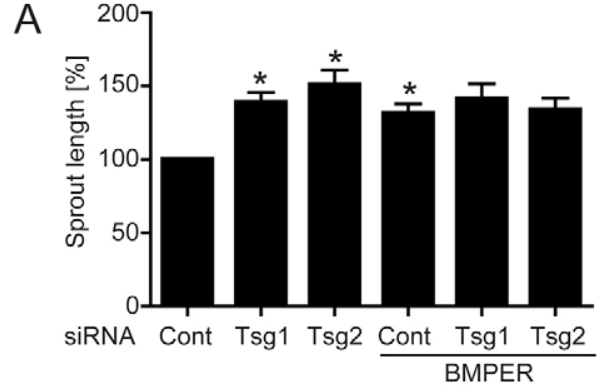

C
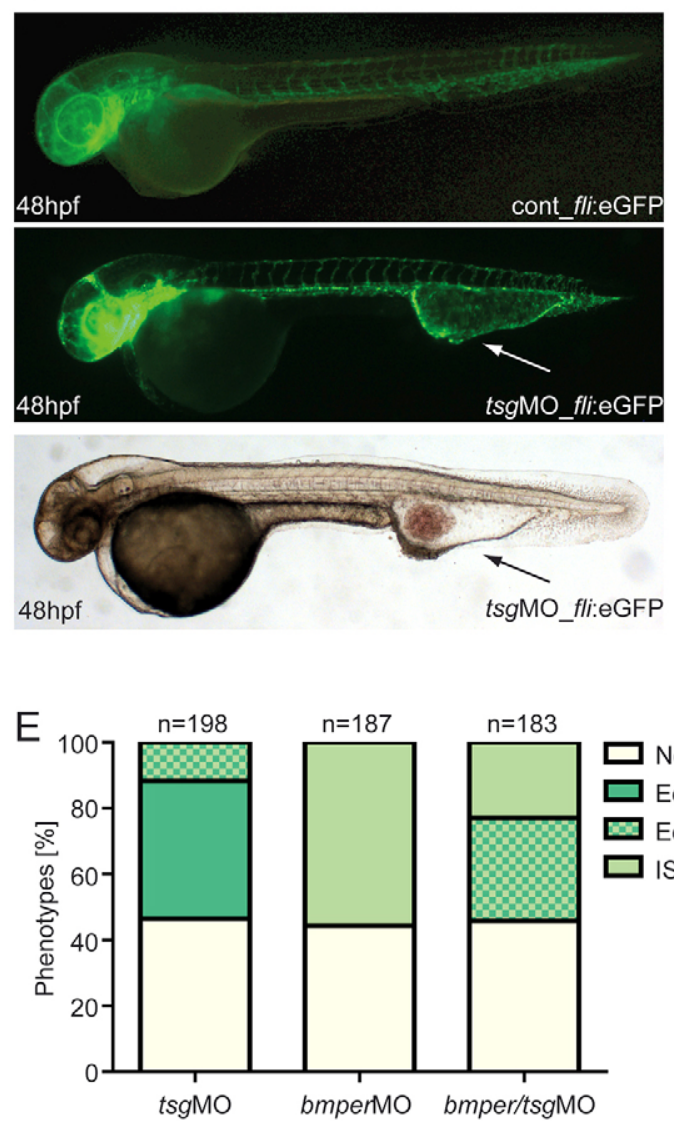

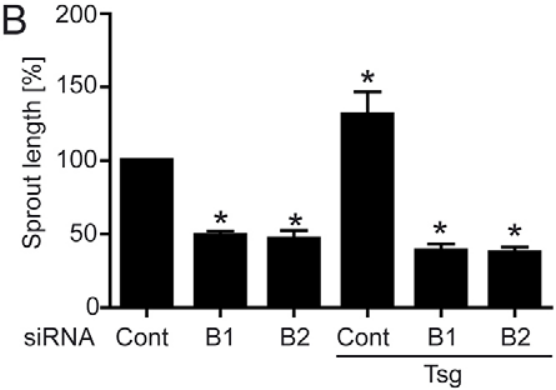

D
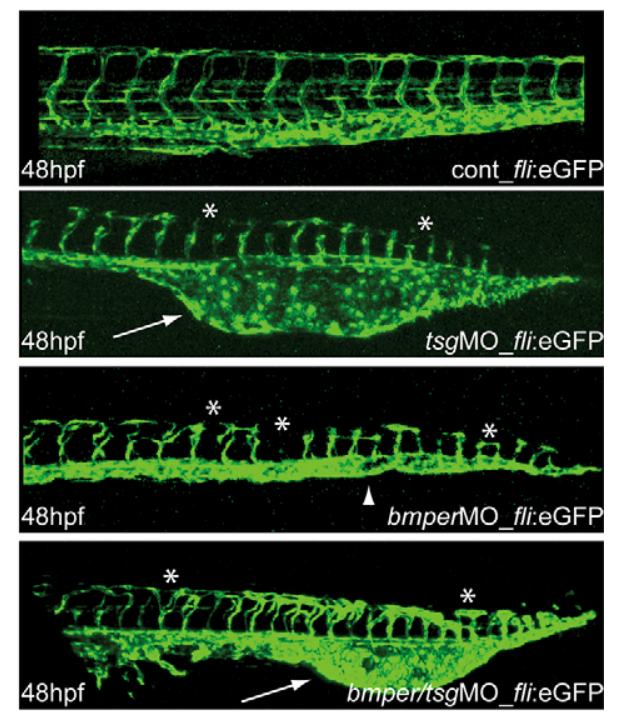

$\square$ Normal

Ectasia

$\square$ Ectasia \& ISV malformation

ISV malformation
Fig. 8. The BMP modulators BMPER and Tsg are necessary for proper angiogenesis in vitro and in zebrafish development. HUVECs were transfected with either Tsg- or BMPER-specific siRNAs, or with scrambled siRNA as a control. Thereafter, Tsg- or BMPERsilenced HUVECs were stimulated with BMPER and Tsg, respectively, and subjected to the tube formation assay. (A) Cumulative sprout length of Tsgsilenced HUVECs stimulated with BMPER and (B) cumulative sprout length of BMPER-silenced HUVECs stimulated with Tsg. Values are means \pm s.e.m.; $n=3$; $* P<0.01$ versus siRNA control. (C-E) Silencing of Tsg in zebrafish embryos causes malformations during blood vessel development. (C) Vasculature morphology of uninjected (top) and $0.125 \mathrm{mM}$ Tsg MO-injected (middle) $\operatorname{tg}$ (flil:eGFP) fish embryos at $48 \mathrm{hpf}$ with formation of a large venous malformation (white arrows). (Bottom) Overall morphology of Tsg MO-injected zebrafish at $48 \mathrm{hpf}$ with blood accumulation in the ventral tail vein ectasia (black arrow). (D) Formation of the trunk vasculature in 48 hpf $\operatorname{tg}(f l i 1: e G F P)$ fish embryos after injection of $0.125 \mathrm{mM}$ Tsg or $0.25 \mathrm{mM}$ BMPER MO or a combination of both. Tsg morphants displayed ventral tail vein ectasia (white arrows) and some disruption of ISVs and the DLAV (asterisks). In BMPER morphants disruption of ISVs and DLAV was more pronounced and in addition loss of the CVP (arrowhead) was observed. Double morphants displayed the malformations of both single Tsg/BMPER morphants. (E) Quantification of vascular defects in $48 \mathrm{hpf} \operatorname{tg}(f l i 1: e G F P) \mathrm{Tsg}$ and BMPER morphants. CVP, caudal vein plexus; DLAV, dorsal longitudinal anastomotic vessel; ISV, intersomitic vessels; hpf, hours post-fertilisation; MO, morpholino oligonucleotide.
In conclusion, extracellular BMP-binding modulators exert different effects on endothelial cell function and angiogenesis. While noggin and chordin had no stimulatory effects, gremlin, BMPER and Tsg enhanced endothelial cell sprouting. For Tsg we observed stimulatory effects on endothelial cells in gain- or lossof-function experiments that appeared to be dependent on the interaction with BMPER. However, in vivo the effect of Tsg on the BMP signalling pathway seems to be highly context and concentration dependent. Altogether, our data indicate that a finetuned equilibrium of Tsg and BMPER controls BMP pathway activity, which is necessary for endothelial cell function.

\section{Materials and methods}

\section{Cell culture and reagents}

Isolation of human umbilical vein endothelial cells (HUVECs) was performed as previously described (Heinke et al., 2008). HUVECs were cultured in enhanced endothelial cell growth medium (PELOBiotech $\mathrm{GmbH}$, Martinsried, Germany). Human coronary artery ECs (HCAEC), heart and lung microvascular ECs (HMECs) were purchased and cultured in EGM2-MV BulletKit medium from Lonza
(Basel, Switzerland). Recombinant mouse Tsg, human BMPER, mouse chordin, human noggin, mouse gremlin, human VEGF-165 and human FGF basic 146 aa protein were reconstituted according to the manufacturer's protocol (R\&D Systems GmbH, Wiesbaden, Germany). MEK1 inhibitor PD98059 and MEK1/2 inhibitor U0126 (also called Erk inhibitors) were reconstituted according to the manufacturer's instructions (Cell Signaling Technology, Danvers, USA). For cell tracking, CFDA-SE (green) and CellTracker ${ }^{\mathrm{TM}}$ Red CMPTX were used (Life Technologies, Karlsruhe, Germany).

\section{Tube formation assay}

Tube formation assay - or Matrigel sprouting assay - was performed as described previously (Heinke et al., 2008). Briefly, HUVECs were pre-treated with the indicated concentration of recombinant proteins in 1\% FBS/EBM for 16-18 hours. Duplets of $2 \times 10^{4}$ cells per condition were cultured on Matrigel with $2 \%$ FBS (BD Biosciences, Heidelberg, Germany) for 3 hours at $37^{\circ} \mathrm{C}, 5 \% \mathrm{CO}_{2}$. Cells were fixed with $4 \%$ paraformaldehyde (PFA) and pictures were taken from four random microscopic fields at $5 \times$ magnification using a digitized imaging system. The cumulative sprout length and the number of branch points were measured with AxioVision Rel. 4.8.

\section{Migration assay}

Cell migration assay was performed as previously described (Heinke et al., 2008) In brief, HUVECs were labelled with $10 \mu \mathrm{M}$ CFDA-SE (Life Technologies), harvested by centrifugation, resuspended in migration medium (RPMI with $0.5 \%$ 
FBS, $0.1 \%$ BSA), counted and placed in the upper chamber of a modified Boyden chamber $\left(1 \times 10^{5}\right.$ cells per HTS FluoroBlok 24 -well chamber; pore size $8 \mu \mathrm{m}$; BD Biosciences). The chambers were placed in 24-well culture dishes containing migration medium and indicated recombinant proteins. After incubation for 4 hours at $37^{\circ} \mathrm{C}, 5 \% \mathrm{CO}_{2}$ the cells were fixed with $4 \%$ PFA and migrated cells were counted manually in five random microscopic fields using a fluorescent microscope.

\section{Proliferation assay}

Proliferation was assessed using a colorimetric BrdU-incorporation ELISA (Roche, Basel, Switzerland) as described recently (Heinke et al., 2012a). In brief, 24 hours after siRNA transfection, cells were cultured in fresh BrdUcontaining $1 \%$ FBS/EBM medium for another 24 hours. For growth factor stimulation cells were directly cultured with BrdU-containing medium for 24 hours. The colorimetric ELISA for BrdU quantification was performed following the manufacturer's instruction.

\section{HUVEC spheroid sprouting assay}

HUVEC spheroid sprouting assay was performed as previously described (Heinke et al., 2008). For mosaic spheroid sprouting experiments siRNA-transfected HUVECs were labelled with CFDA-SE (green) or CMPTX (red), respectively and mixed in equal amounts before hanging drops were generated. Collagen embedded mosaic spheroids were imaged with an inverted ZEISS LSM 5 Live DUO high speed confocal microscope at the Life Imaging Center, ZBSA, Freiburg, Germany.

\section{Mouse Matrigel plug assay and immunohistochemistry}

Experiments were performed according to the Animals Scientific Procedures Act of 1986 and local ethics protocols. Mouse Matrigel plug assay was performed as described previously (Heinke et al., 2008). In brief, Matrigel (BD Biosciences) was mixed with heparin and recombinant proteins and injected subcutaneously into the abdominal flanks of female C57BL/6N mice (Charles River, Sulzfeld, Germany). After 10 days, plugs were isolated, fixed in 4\% PFA and sectioned. To determine endothelial cell specificity slides were stained with anti-CD31 (BD Pharmingen) and goat anti-rat $\mathrm{Cy}-3$ antibodies (Chemicon International). Blood vessel infiltration was analysed in 10 random Haematoxylin- and Eosin-stained sections imaged with Zeiss Axioplan2/Axiovision Rel. 4.8.

\section{Western blot analysis}

Western blot analysis was performed as previously described (Heinke et al., 2008). Primary antibodies were incubated overnight at $4{ }^{\circ} \mathrm{C}$ in $3 \%$ non-fat dried milk/ TBST: anti-mouse Tsg (monoclonal rat ab; 1:1000; R\&D Systems); anti-human BMPER (monoclonal rat ab; 1:3000; R\&D Systems); anti-human $\beta$-tubulin (monoclonal mouse ab; 1:3000; R\&D Systems); anti-P-Akt1/2, P-Erk1/2, PSmad1/5, Erk1/2 and Smad5 (polyclonal rabbit abs; 1:1000; Cell Signaling Technologies); anti-Gapdh (polyclonal rabbit ab; EnoGene, New York, USA).

Secondary antibodies conjugated to horseradish peroxidase were incubated for 1-2 hours at room temperature in 3\% non-fat dried milk/TBST: anti-mouse-HRP (polyclonal ab, 1:5000; R\&D Systems); anti-rabbit-HRP (polyclonal ab, 1:10000, Thermo Fisher Scientific); anti-rat-HRP (polyclonal ab, 1:5000; Dako).

Visualisation was performed using an ECL system (Amersham Bioscience) and a digital imaging system (chemiDOC XRS and Image Lab 4.0; Bio-Rad).

\section{RNA interference}

BMPRII, Tsg and BMPER siRNAs 1 and 2 were purchased from Life Technologies, respectively. Scrambled negative control Alexa Fluor 488 was purchased from Qiagen, Hilden, Germany. For transfection a final concentration of $100 \mathrm{nmol} / 1$ siRNA together with Lipofectamine RNAiMAX was used according to the manufacturer's protocol (Life Technologies). Transfection efficiency was confirmed by quantitative real-time (q) PCR. Functional cell culture assays were performed between 8 and 48 hours post transfection.

siRNA sequences: siTsg 1, forward 5'-CGCAUGUUUCCUGGGAGUUACUGAU-3', reverse 5'-AUCAGUAACUCCCAGGAAACAUGCG-3'; siTsg 2, forward 5'-CAUCCUGAUGUUCCUGACAUGGCUU-3', reverse 5'-AAGCCAUGUCAGGAACAUCAGGAUG-3'; siBMPRII 1, forward 5'-GCUGUUGUAGCACAGAUUUAUGUAA- ${ }^{\prime}$, reverse 5'-UUACAUAAAUCUGUGCUACAACAGC-3'; siBMPRII 2, forward 5'-GCCUUUGAUGGAACAUGACAACAUU-3', reverse 5'-AAUGUUGUCAUGUUCCAUCAAAGGC-3'; siBMPER 1, forward 5'-GCACCUUAGUCACAUACCCTT- ${ }^{\prime}$, reverse $5^{\prime}$-GGGUAUGUGACUAAGGUGCTG-3'; siBMPER 2, forward 5'-GCUGCCUCUUUCGAAGUGATT-3', reverse 5'-UCACUUCGAAAGAGGCAGCTC-3'.

\section{Immunocytofluorescence}

HUVECs grown on glass coverslips were fixed first with 4\% PFA and then blocked with $10 \%$ goat serum for 30 minutes at room temperature. Afterwards cells were incubated over night at $4^{\circ} \mathrm{C}$ with the monoclonal Tsg antibody $(1: 100$; R\&D Systems) or with control ratIgG2a (BD Pharmingen, Heidelberg, Germany), respectively. The staining was completed with goat-anti rat-Cy3 (1:500; Chemicon
International). For visualization of nuclei slides were treated with DAPI (1:30000; Sigma, Deisenhofen, Germany). All photographs were taken with Zeiss Axioplan2 and analysed with Zeiss Axiovision Rel. 4.8.

\section{RNA extraction and reverse transcription}

DNA-free total RNA was extracted from HUVEC, HCAEC, HMEC lung and HMEC heart using the Aurum RNA Mini Kit (Bio-Rad, Munich, Germany). Reverse transcriptions were performed with iScript cDNA-Kit applying $1 \mu \mathrm{g}$ RNA following the manufacturer's protocol (Bio-Rad).

\section{Semi-quantitative and real-time PCR}

Reverse transcription-PCR (RT-PCR) analysis was performed as described previously (Heinke et al., 2008).

Quantitative real-time PCR analysis following RNA interference was performed using IQ SybrGreen 2xSupermix and the iCycler real-time PCR detection system (Bio-Rad). Quantification was performed using MyiQ lightcycler software (BioRad). Knockdown efficiency was calculated using the $\Delta \Delta \mathrm{C}_{\mathrm{T}}$ method (Schmittgen and Livak, 2008; Wong and Medrano, 2005). The housekeeping gene hRP was used for internal normalization.

Primer sequences: Tsg, forward primer $5^{\prime}$-ACTCTAGCCATCCTGATGTTCC3', reverse primer 5'-CAACACAGTCACAGCACTCG-3'; BMPER, forward primer 5'-AGGACAGTGCTGCCCCAAATG-3', reverse primer 5'-TACTGACACGTCCCCTGAAAG-3'; BMPRII, forward primer 5'-ACCAGAAGTGCTAGAAGGAG-3', reverse primer 5'-GCCGAGCCTCTGCATCCTGGT-3'; hRPII, forward primer 5'-GCACCACGTCCAATGACA T-3', reverse primer 5'-GTGCGGCTGCTTCCATAA-3'.

\section{Fish strains, morpholino and RNA injections}

Zebrafish embryos of the $\operatorname{tg}(f l i 1: e G F P$ ) line (Lawson and Weinstein, 2002) were raised (Westerfield, 1995) and staged as described (Kimmel et al., 1995). Fertilized eggs were kept in $0.3 \times$ Danieau's solution at $28^{\circ} \mathrm{C}$ with addition of $0.003 \% 1$ phenyl-2-thiourea (Sigma-Aldrich, Taufkirchen, Germany) at 24 hours postfertilisation to suppress pigmentation.

For morpholino oligonucleotide (MO) injections one or two cell stage embryos were injected with $1 \mathrm{nl}$ of the indicated amounts of MOs and raised at $28^{\circ} \mathrm{C}$ until analysed. We used previously described MOs targeted against Tsg (Ross et al., 2001) and $z B M P E R$ MO1 (Moser et al., 2007) (Gene Tools, LLC, Philomath, USA).

\section{Statistical analysis and quantification}

Statistical analysis was performed using GraphPad Prism 5.0, La Jolla, USA. Data are presented as means \pm s.e.m. and comparisons were calculated by Student's $t$ test (two-way, unpaired). All experiments were repeated at least three times in triplicates. Results were considered statistically significant if $P<0.05$.

\section{Acknowledgements}

We are indebted to Bianca Engert and Ute Wering for excellent technical assistance. We are grateful to the group of Albrecht Kramer-Zucker, Nephro Lab, UFMC, for excellent help with zebrafish experiments. We thank Wolfgang Driever for sharing the transgenic zebrafish line $\operatorname{tg}(f l i 1: e G F P)$ and the staff of the Life Imaging Center (LIC), Center for Biological Systems Analysis (all at the University of Freiburg).

\section{Author contributions}

J.H. designed and performed the experiments, analyzed the data and wrote the paper. M.J., L.M., and A.C. performed the experiments. A.C., T.H. and C.B. analyzed the data. C.P. wrote the paper and M.M. planned and supervised the project and wrote the paper.

\section{Funding}

This work was supported by the German Research Foundation [grant numbers DFG Mo973/5-1, DFG Mo973/6-1 to M.M.]; and the University of Freiburg intramural funds (M.M.'s laboratory).

Supplementary material available online at http://jcs.biologists.org/lookup/suppl/doi:10.1242/jcs.122333/-/DC1

\section{References}

Abreu, J. G., Ketpura, N. I., Reversade, B. and De Robertis, E. M. (2002). Connective-tissue growth factor (CTGF) modulates cell signalling by BMP and TGFbeta. Nat. Cell Biol. 4, 599-604.

Adams, R. H. and Alitalo, K. (2007). Molecular regulation of angiogenesis and lymphangiogenesis. Nat. Rev. Mol. Cell Biol. 8, 464-478. 
Ambrosio, A. L., Taelman, V. F., Lee, H. X., Metzinger, C. A., Coffinier, C. and De Robertis, E. M. (2008). Crossveinless-2 Is a BMP feedback inhibitor that binds Chordin/BMP to regulate Xenopus embryonic patterning. Dev. Cell 15, 248-260.

Balemans, W. and Van Hul, W. (2002). Extracellular regulation of BMP signaling in vertebrates: a cocktail of modulators. Dev. Biol. 250, 231-250.

Bier, E. (2008). Intriguing extracellular regulation of BMP signaling. Dev. Cell 15, 176 177.

Bragdon, B., Moseychuk, O., Saldanha, S., King, D., Julian, J. and Nohe, A. (2011) Bone morphogenetic proteins: a critical review. Cell. Signal. 23, 609-620.

Carmeliet, P. and Jain, R. K. (2011). Molecular mechanisms and clinical applications of angiogenesis. Nature 473, 298-307.

Chang, C., Holtzman, D. A., Chau, S., Chickering, T., Woolf, E. A., Holmgren, L. M., Bodorova, J., Gearing, D. P., Holmes, W. E. and Brivanlou, A. H. (2001) Twisted gastrulation can function as a BMP antagonist. Nature 410, 483-487.

Chiodelli, P., Mitola, S., Ravelli, C., Oreste, P., Rusnati, M. and Presta, M. (2011) Heparan sulfate proteoglycans mediate the angiogenic activity of the vascular endothelial growth factor receptor-2 agonist gremlin. Arterioscler. Thromb. Vasc. Biol. 31, e116-e127.

David, L., Feige, J. J. and Bailly, S. (2009). Emerging role of bone morphogenetic proteins in angiogenesis. Cytokine Growth Factor Rev. 20, 203-212.

Heinke, J., Wehofsits, L., Zhou, Q., Zoeller, C., Baar, K. M., Helbing, T., Laib, A. Augustin, H., Bode, C., Patterson, C. et al. (2008). BMPER is an endothelial cell regulator and controls bone morphogenetic protein-4-dependent angiogenesis. Circ. Res. 103, 804-812.

Heinke, J., Kerber, M., Rahner, S., Mnich, L., Lassmann, S., Helbing, T., Werner, M., Patterson, C., Bode, C. and Moser, M. (2012a). Bone morphogenetic protein modulator BMPER is highly expressed in malignant tumors and controls invasive cell behavior. Oncogene 31, 2919-2930.

Heinke, J., Patterson, C. and Moser, M. (2012b). Life is a pattern: vascular assembly within the embryo. Front. Biosci. (Elite Ed.) 4, 2269-2288.

Helbing, T., Rothweiler, R., Heinke, J., Goetz, L., Diehl, P., Zirlik, A., Patterson, C. Bode, C. and Moser, M. (2010). BMPER is upregulated by statins and modulates endothelial inflammation by intercellular adhesion molecule-1. Arterioscler. Thromb. Vasc. Biol. 30, 554-560.

Helbing, T., Rothweiler, R., Ketterer, E., Goetz, L., Heinke, J., Grundmann, S., Duerschmied, D., Patterson, C., Bode, C. and Moser, M. (2011). BMP activity controlled by BMPER regulates the proinflammatory phenotype of endothelium. Blood 118, 5040-5049.

Herbert, S. P. and Stainier, D. Y. (2011). Molecular control of endothelial cell behaviour during blood vessel morphogenesis. Nat. Rev. Mol. Cell Biol. 12, 551-564.

Herrera, B. and Inman, G. J. (2009). A rapid and sensitive bioassay for the simultaneous measurement of multiple bone morphogenetic proteins. Identification and quantification of BMP4, BMP6 and BMP9 in bovine and human serum. BMC Cell Biol. 10, 20.

Horowitz, A. and Simons, M. (2008). Branching morphogenesis. Circ. Res. 103, 784 795.

Ikeya, M., Nosaka, T., Fukushima, K., Kawada, M., Furuta, Y., Kitamura, T. and Sasai, Y. (2008). Twisted gastrulation mutation suppresses skeletal defect phenotypes in Crossveinless 2 mutant mice. Mech. Dev. 125, 832-842.

Ikeya, M., Fukushima, K., Kawada, M., Onishi, S., Furuta, Y., Yonemura, S., Kitamura, T., Nosaka, T. and Sasai, Y. (2010). Cv2, functioning as a pro-BMP factor via twisted gastrulation, is required for early development of nephron precursors. Dev. Biol. 337, 405-414.

Kelley, R., Ren, R., Pi, X., Wu, Y., Moreno, I., Willis, M., Moser, M., Ross, M. Podkowa, M., Attisano, L. et al. (2009). A concentration-dependent endocytic trap and sink mechanism converts Bmper from an activator to an inhibitor of Bmp signaling. J. Cell Biol. 184, 597-609.

Khoo, C. P., Micklem, K. and Watt, S. M. (2011). A comparison of methods for quantifying angiogenesis in the Matrigel assay in vitro. Tissue Eng. Part C Methods 17, 895-906

Kimmel, C. B., Ballard, W. W., Kimmel, S. R., Ullmann, B. and Schilling, T. F. (1995). Stages of embryonic development of the zebrafish. Dev. Dyn. 203, 253-310.

Lawson, N. D. and Weinstein, B. M. (2002). In vivo imaging of embryonic vascular development using transgenic zebrafish. Dev. Biol. 248, 307-318.

Lillis, A. P., Van Duyn, L. B., Murphy-Ullrich, J. E. and Strickland, D. K. (2008) LDL receptor-related protein 1: unique tissue-specific functions revealed by selective gene knockout studies. Physiol. Rev. 88, 887-918.

Little, S. C. and Mullins, M. C. (2004). Twisted gastrulation promotes BMP signaling in zebrafish dorsal-ventral axial patterning. Development 131, 5825-5835.

Lugus, J. J., Chung, Y. S., Mills, J. C., Kim, S. I., Grass, J., Kyba, M., Doherty, J. M., Bresnick, E. H. and Choi, K. (2007). GATA2 functions at multiple steps in hemangioblast development and differentiation. Development 134, 393-405.

Marshall, C. J., Kinnon, C. and Thrasher, A. J. (2000). Polarized expression of bone morphogenetic protein-4 in the human aorta-gonad-mesonephros region. Blood $\mathbf{9 6}$ 1591-1593.

Martin, P. and Parkhurst, S. M. (2004). Parallels between tissue repair and embryo morphogenesis. Development 131, 3021-3034.

Mitola, S., Ravelli, C., Moroni, E., Salvi, V., Leali, D., Ballmer-Hofer, K., Zammataro, L. and Presta, M. (2010). Gremlin is a novel agonist of the major proangiogenic receptor VEGFR2. Blood 116, 3677-3680.

Moreno-Miralles, I., Schisler, J. C. and Patterson, C. (2009). New insights into bone morphogenetic protein signaling: focus on angiogenesis. Curr. Opin. Hematol. 16, 195-201.
Moreno-Miralles, I., Ren, R., Moser, M., Hartnett, M. E. and Patterson, C. (2011) Bone morphogenetic protein endothelial cell precursor-derived regulator regulates retinal angiogenesis in vivo in a mouse model of oxygen-induced retinopathy. Arterioscler. Thromb. Vasc. Biol. 31, 2216-2222.

Moser, M., Binder, O., Wu, Y., Aitsebaomo, J., Ren, R., Bode, C., Bautch, V. L. Conlon, F. L. and Patterson, C. (2003). BMPER, a novel endothelial cell precursorderived protein, antagonizes bone morphogenetic protein signaling and endothelial cell differentiation. Mol. Cell. Biol. 23, 5664-5679.

Moser, M., Yu, Q., Bode, C., Xiong, J. W. and Patterson, C. (2007). BMPER is a conserved regulator of hematopoietic and vascular development in zebrafish. J. Mol. Cell. Cardiol. 43, 243-253

Moya, I. M., Umans, L., Maas, E., Pereira, P. N., Beets, K., Francis, A., Sents, W., Robertson, E. J., Mummery, C. L., Huylebroeck, D. et al. (2012). Stalk cell phenotype depends on integration of Notch and Smad1/5 signaling cascades. Dev. Cell 22, 501-514.

Muñoz-Chápuli, R., Quesada, A. R. and Angel Medina, M. (2004). Angiogenesis and signal transduction in endothelial cells. Cell. Mol. Life Sci. 61, 2224-2243.

Muratoglu, S. C., Mikhailenko, I., Newton, C., Migliorini, M. and Strickland, D. K. (2010). Low density lipoprotein receptor-related protein 1 (LRP1) forms a signaling complex with platelet-derived growth factor receptor-beta in endosomes and regulates activation of the MAPK pathway. J. Biol. Chem. 285, 14308-14317.

Nimmagadda, S., Geetha Loganathan, P., Huang, R., Scaal, M., Schmidt, C. and Christ, B. (2005). BMP4 and noggin control embryonic blood vessel formation by antagonistic regulation of VEGFR-2 (Quek1) expression. Dev. Biol. 280, 100-110.

Nosaka, T., Morita, S., Kitamura, H., Nakajima, H., Shibata, F., Morikawa, Y., Kataoka, Y., Ebihara, Y., Kawashima, T., Itoh, T. et al. (2003). Mammalian twisted gastrulation is essential for skeleto-lymphogenesis. Mol. Cell. Biol. 23, 29692980

Oelgeschläger, M., Larraín, J., Geissert, D. and De Robertis, E. M. (2000). The evolutionarily conserved BMP-binding protein Twisted gastrulation promotes BMP signalling. Nature 405, 757-763.

Olsson, A. K., Dimberg, A., Kreuger, J. and Claesson-Welsh, L. (2006). VEGF receptor signalling - in control of vascular function. Nat. Rev. Mol. Cell Biol. 7, 359371.

Pi, X., Lockyer, P., Dyer, L. A., Schisler, J. C., Russell, B., Carey, S., Sweet, D. T., Chen, Z., Tzima, E., Willis, M. S. et al. (2012a). Bmper inhibits endothelia expression of inflammatory adhesion molecules and protects against atherosclerosis. Arterioscler. Thromb. Vasc. Biol. 32, 2214-2222.

Pi, X., Schmitt, C. E., Xie, L., Portbury, A. L., Wu, Y., Lockyer, P., Dyer, L. A., Moser, M., Bu, G., Flynn, E. J. et al. (2012b). An LRP1-dependent endocytic mechanism governs the signaling output of the bmp system in endothelial cells and in angiogenesis. Circ. Res. 111, 564-574.

Piccolo, S., Sasai, Y., Lu, B. and De Robertis, E. M. (1996). Dorsoventral patterning in Xenopus: inhibition of ventral signals by direct binding of chordin to BMP-4. Cell $\mathbf{8 6}$, 589-598.

Ponce, M. L. (2009). Tube formation: an in vitro matrigel angiogenesis assay. Methods Mol. Biol. 467, 183-188

Potente, M., Gerhardt, H. and Carmeliet, P. (2011). Basic and therapeutic aspects of angiogenesis. Cell 146, 873-887.

Reese, D. E., Hall, C. E. and Mikawa, T. (2004). Negative regulation of midline vascular development by the notochord. Dev. Cell 6, 699-708.

Risau, W. and Flamme, I. (1995). Vasculogenesis. Annu. Rev. Cell Dev. Biol. 11, 73 91

Ross, J. J., Shimmi, O., Vilmos, P., Petryk, A., Kim, H., Gaudenz, K., Hermanson, S., Ekker, S. C., O'Connor, M. B. and Marsh, J. L. (2001). Twisted gastrulation is a conserved extracellular BMP antagonist. Nature 410, 479-483.

Scharpfenecker, M., van Dinther, M., Liu, Z., van Bezooijen, R. L., Zhao, Q., Pukac, L., Löwik, C. W. and ten Dijke, P. (2007). BMP-9 signals via ALK1 and inhibits bFGF-induced endothelial cell proliferation and VEGF-stimulated angiogenesis. J. Cell Sci. 120, 964-972.

Schmierer, B. and Hill, C. S. (2007). TGFbeta-SMAD signal transduction: molecular specificity and functional flexibility. Nat. Rev. Mol. Cell Biol. 8, 970-982.

Schmittgen, T. D. and Livak, K. J. (2008). Analyzing real-time PCR data by the comparative C(T) method. Nat. Protoc. 3, 1101-1108.

Scott, I. C., Blitz, I. L., Pappano, W. N., Maas, S. A., Cho, K. W. and Greenspan, D. S. (2001). Homologues of Twisted gastrulation are extracellular cofactors in antagonism of BMP signalling. Nature 410, 475-478.

Serpe, M., Umulis, D., Ralston, A., Chen, J., Olson, D. J., Avanesov, A., Othmer, H., O'Connor, M. B. and Blair, S. S. (2008). The BMP-binding protein Crossveinless 2 is a short-range, concentration-dependent, biphasic modulator of BMP signaling in Drosophila. Dev. Cell 14, 940-953.

Shiojima, I. and Walsh, K. (2002). Role of Akt signaling in vascular homeostasis and angiogenesis. Circ. Res. 90, 1243-1250.

Sieber, C., Kopf, J., Hiepen, C. and Knaus, P. (2009). Recent advances in BMP receptor signaling. Cytokine Growth Factor Rev. 20, 343-355.

Smith, W. C. and Harland, R. M. (1992). Expression cloning of noggin, a new dorsalizing factor localized to the Spemann organizer in Xenopus embryos. Cell 70, 829-840.

Sonnemann, K. J. and Bement, W. M. (2011). Wound repair: toward understanding and integration of single-cell and multicellular wound responses. Annu. Rev. Cell Dev. Biol. 27, 237-263. 
Stabile, H., Mitola, S., Moroni, E., Belleri, M., Nicoli, S., Coltrini, D., Peri, F., Pessi, A., Orsatti, L., Talamo, F. et al. (2007). Bone morphogenic protein antagonist Drm/ gremlin is a novel proangiogenic factor. Blood 109, 1834-1840.

ten Dijke, P. and Arthur, H. M. (2007). Extracellular control of TGFbeta signalling in vascular development and disease. Nat. Rev. Mol. Cell Biol. 8, 857-869.

Umulis, D., O'Connor, M. B. and Blair, S. S. (2009). The extracellular regulation of bone morphogenetic protein signaling. Development 136, 3715-3728.

Wagner, D. O., Sieber, C., Bhushan, R., Börgermann, J. H., Graf, D. and Knaus, P. (2010). BMPs: from bone to body morphogenetic proteins. Sci. Signal. 3, mr1.

Westerfield, M. (1995). The Zebrafish Book: A Guide for the Laboratory Use of Zebrafish (Danio rerio). University of Oregon Press, Eugene.

Wiley, D. M., Kim, J. D., Hao, J., Hong, C. C., Bautch, V. L. and Jin, S. W. (2011). Distinct signalling pathways regulate sprouting angiogenesis from the dorsal aorta and the axial vein. Nat. Cell Biol. 13, 686-692.
Wong, M. L. and Medrano, J. F. (2005). Real-time PCR for mRNA quantitation. Biotechniques 39, 75-85.

Xie, J. and Fisher, S. (2005). Twisted gastrulation enhances BMP signaling through chordin dependent and independent mechanisms. Development 132, 383-391.

Yamamoto, Y. and Oelgeschläger, M. (2004). Regulation of bone morphogenetic proteins in early embryonic development. Naturwissenschaften 91, 519-534.

Zakin, L. and De Robertis, E. M. (2004). Inactivation of mouse Twisted gastrulation reveals its role in promoting Bmp4 activity during forebrain development. Development 131, 413-424.

Zakin, L., Metzinger, C. A., Chang, E. Y., Coffinier, C. and De Robertis, E. M. (2008). Development of the vertebral morphogenetic field in the mouse: interactions between Crossveinless-2 and Twisted Gastrulation. Dev. Biol. 323, 6-18.

Zhou, Q., Heinke, J., Vargas, A., Winnik, S., Krauss, T., Bode, C., Patterson, C. and Moser, M. (2007). ERK signaling is a central regulator for BMP-4 dependent capillary sprouting. Cardiovasc. Res. 76, 390-399. 